\title{
Estratégias para Aplicação no Trabalho do Aprendido em Treinamento: Proposição Conceitual e Desenvolvimento de uma Medida
}

\author{
Ronaldo Pilati ${ }^{1}$ \\ Instituto de Ciências do Trabalho e UNICEUB \\ Jairo E. Borges-Andrade ${ }^{2}$ \\ Universidade de Brasília
}

\begin{abstract}
Resumo
O papel exercido pelo treinando no ambiente posterior ao treinamento ainda é pouco explorado na literatura especializada, enquanto vários modelos de avaliação de impacto do treinamento no trabalho ressaltam o papel exercido pelas condições ambientais no contexto póstreinamento. Esse trabalho pretende explorar aquele papel, tendo como objetivo propor um conceito para o estudo do fenômeno e validar uma medida para sua mensuração. Foi realizada uma análise conceitual do fenômeno e uma escala para sua mensuração foi desenvolvida. A escala foi aplicada em uma amostra de trabalhadores e os dados submetidos a análises para aferição da estrutura latente. Os resultados corroboram os pressupostos teóricos da análise conceitual, mas devem ser realizados mais estudos para: a) descrever melhor as características do fenômeno; e, b) relacioná-lo com indicadores de resultado do treinamento no trabalho.

Palavras-chave: Avaliação de treinamento; estratégias para aplicação no trabalho do aprendido em treinamento; contexto pós-treinamento.
\end{abstract}

\section{Work Application Strategies of Learning from Training: Conceptual Proposition and Development of a Measure}

\begin{abstract}
The role of the ex-trainee in the post training environment is not enough studied, while several evaluation models of training impact highlight the role of contextual variables at the post training environment. This work intends to investigate that role. The objectives are to propose a concept for studying the phenomenon and to validate a questionnaire for its measurement. A conceptual analysis of the construct has been done and a scale has been developed. This scale was applied in a sample of workers and the data was analyzed by using a latent structure technique. The results corroborate the theoretical presuppositions of the conceptual analysis, but more studies need to be done to a) better describe the construct characteristics; and b) relate it with indicators of training results at work.

Keywords: Training evaluation; work application strategies of learning from training; post-training context.
\end{abstract}

A área de avaliação de treinamento e desenvolvimento (T\&D) de pessoal tem como um dos principais focos de interesse a transferência de aprendizagem e o impacto do treinamento no trabalho das pessoas. $\mathrm{O}$ investimento que as organizações fazem com treinamento de pessoal é considerável e a necessidade de aferir que tipo de resultado o treinamento provoca na ocupação dos trabalhadores torna-se condição necessária para avaliar se os recursos investidos estão realmente surtindo o efeito desejado (Holton III, Bates, Seyler \& Carvalho, 1997). Além disso, é fundamental compreender quais variáveis efetivamente afetam o resultado do treinamento no trabalho, para que os responsáveis possam intervir no sistema de treinamento visando torná-lo mais efetivo.

Foram desenvolvidos muitos modelos para compreender que fatores levam ao impacto do treinamento no trabalho (Ex.: Abbad, 1999; Borges-Andrade, 1982; Goldstein, 1993; Sallorenzo, 2000; Tannenbaum, Mathieu, Salas \& Cannon-Bowers, 1991). As variáveis que compõem esses modelos podem ser divididas em três grandes categorias: instrucionais, contextuais e individuais, conforme uma revisão de literatura feita por Abbad, Pilati e Pantoja (2003). As primeiras dizem respeito ao planejamento instrucional e execução do treinamento. Um conjunto de evidências mais antigo aponta para a importância dessas variáveis como preditoras do impacto do

${ }^{1}$ Esse trabalho teve apoio da CAPES e do PRONEX - Treinamento e Comportamento no Trabalho. Os autores agradecem a colaboração, essencial em todas as etapas de coleta de dados, de Paulo de Tarço da Silva, Fátima Bruno de Faria, Helena Tonet, Pricila Sabino, Isa Freitas, Hugo Brandão e Marcos Fadanelli.

${ }^{2}$ Endereço para correspondência: Universidade de Brasilia, Instituto de Psicologia, Departamento de Psicologia Social e do Trabalho, 70910 900, Brasilia, DF. Fone: (61) 3072625 (Ramal 221); Fax: (61) 2730203.E-mail:jeborges@linkexpress.com.br treinamento no trabalho, como foi ressaltado na revisão de literatura de Borges-Andrade e Abbad-OC (1996).

As variáveis contextuais dizem respeito ao conjunto de fatores externos ao contexto de treinamento, presentes no ambiente organizacional, que influenciam a transferência do aprendido. Mais recentemente, estudos (Ex.: Abbad, Borges-Andrade, Sallorenzo, Gama \& Morandini, 2001; Abbad, Gama \& Borges-Andrade, 2000; Britto, Lima \& Borges-Andrade, 2001; Holton III \& cols., 1997; Lima, Borges-Andrade \& Vieira, 1989; Roullier \& Goldstein, 1993; Sallorenzo, 2000) têm apontado a importância do suporte organizacional à transferência de treinamento como fator crítico para que o treinamento provoque mudanças no trabalho. As variáveis individuais, relativas à terceira categoria anteriormente mencionada, dizem respeito às características dos treinandos que influenciam o processo de transferência do aprendido para o trabalho. Alguns autores (Ex.: Colquit, LePine \& Noe, 2000; Lacerda, 2002; Warr \& Bunce, 1995) pontuam que variáveis de personalidade e atitudinais influenciam no resultado do treinamento no trabalho dos treinados.

Sem dúvida, a literatura em avaliação de T\&D já caminha para uma convergência dos modelos teóricos com relação ao papel exercido por variáveis contextuais no ambiente pós-treinamento. Revisões nacionais e internacionais (Ex.: respectivamente, Abbad, Pilati \& Pantoja, 2003; Salas \& Cannon-Bowers, 2001) apontam para evidências da influência dessas variáveis sobre o resultado, podendo-se assegurar que treinamentos apenas tendem a ser efetivos se o ambiente posterior ao treinamento oferecer requisitos de suporte (como denomina a literatura nacional) ou clima (de acordo com a literatura internacional) para a transferência do aprendido. 
Apesar dessas evidências empíricas, existe uma questão que necessita ser mais bem estudada no ambiente posterior ao treinamento. Ela diz respeito ao papel do treinado como agente de modificação do ambiente pós-treinamento, para a criação de condições suficientes e necessárias para a aplicação do aprendido no trabalho. Alguns estudos procuram produzir tecnologias para desenvolver essas competências nos treinandos. Essa linha de pesquisa teria como função descrever um conjunto de ações promovidas pelo indivíduo no ambiente de trabalho exatamente para modificá-lo e torná-lo mais receptivo às novas competências. Assim, esses estudos teriam como objetivo descrever a influência de variáveis individuais, de criação de condições de aplicação, no contexto pós-treinamento.

Nessa perspectiva, Marx (1982) apresentou um modelo influente. Ele é denominado de prevenção de recaídas ou auto-gerenciamento e é baseado em um modelo da psicologia clínica desenvolvido para diminuir as recaídas de adictos a drogas. O segundo modelo, bastante difundido, é denominado estabelecimento de metas e tem origem na psicologia organizacional, principalmente na área de avaliação de desempenho. O estabelecimento de metas associado ao conteúdo de um evento instrucional, assim como o treinamento em prevenção de recaídas aplicado a T\&D, são apresentados a seguir.

Segundo Locke, Shaw, Saari e Latham (1981), o conceito de metas é parecido com os de intenção e propósito (ou objetivo), mas é definido pelos autores como aquilo que um indivíduo almeja atingir por meio de uma ação específica. Para eles, as duas dimensões do estabelecimento de metas (i.e., dificuldade e especificidade da meta) são relacionadas ao desempenho no trabalho. Na área de T\&D, Wexley e Baldwin (1986) apresentaram um trabalho no qual compararam duas estratégias de intervenção, posterior ao treinamento, para aumentar a probabilidade de transferência de aprendizagem. Uma dessas estratégias foi a de estabelecimento de metas comportamentais específicas, relacionadas ao conteúdo do treinamento, para aplicação do aprendido no trabalho. Apesar da estratégia de estabelecimento de metas ser há muito tempo utilizada na área de comportamento organizacional, esta passou a ser aplicada a T\&D na década de 1980 (Wexley \& Baldwin, 1986). Vários estudos têm apontado para a efetividade desta estratégia como meio de aumentar a probabilidade de transferência de treinamento (Ex.: Gist, Stevens \& Bavetta, 1991; Stevens \& Gist, 1997; Wexley \& Baldwin, 1986) em diferentes eventos instrucionais, dos mais variados domínios taxonômicos de aprendizagem.

Marx (1982) aplicou o modelo de prevenção de recaída em comportamentos indesejáveis no ambiente pós-treinamento em uma ação instrucional para gerentes. Este modelo baseou-se no trabalho de Marlatt e Gordon (1980, citados em Marx, 1982), desenvolvido na área de reabilitação de pacientes dependentes de drogas. Ele baseia-se no pressuposto de que situações de alto risco de recaída em ambiente posterior ao treinamento (Ex.: pressão temporal) aumentam a probabilidade do gerente não aplicar os conhecimentos, habilidades e atitudes desenvolvidos. Assim, é essencial possibilitar que os gerentes treinados aprendam a identificar e enfrentar as situações de alto risco, facilitando a transferência do aprendido para o ambiente de trabalho.
Apesar do modelo de Marx (1982) ter sua origem no tratamento de pessoas dependentes ou com comportamentos compulsivos e do autor ter adaptado o modelo para treinamento gerencial, a aplicação dele pode ser estendida para muitos outros tipos de treinamentos e clientelas. Vários trabalhos já testaram esse modelo (Ex.: Burke, 1997; Gist \& cols., 1991; Morin \& Latham, 2000; Richman- Hirsch, 2001; Stevens \& Gist, 1997; Wexley \& Baldwin, 1986), indicando o sucesso dele no aumento da transferência de aprendizagem para o trabalho.

A investigação sobre estratégias de enfrentamento e estabelecimento de metas em T\&D somente estiveram interessadas em testar modelos de instrução visando o aumento de transferência do aprendido para o trabalho. Esses modelos geralmente eram módulos à parte nos treinamentos, com o objetivo de ensinar aos treinados maneiras de facilitar a transferência. Esses módulos foram ou baseados em metas ou em prevenção de recaída (Ex.: Burke, 1997; Wexley \& Baldwin, 1986). Não é observado na literatura nenhum esforço que procure identificar um conjunto de ações para a aplicação do aprendido no trabalho desenvolvidos independentemente da instrução, graças à experiência profissional com treinamentos.

Uma variável de comportamento organizacional que está relacionada com as estratégias de aplicação no trabalho do aprendido em treinamento é o comportamento pro-ativo nas organizações. Segundo Crant (2000), a literatura sobre comportamento proativo apresenta uma discussão sobre o que são e como se manifestam esses comportamentos no ambiente de trabalho. São definidos como uma iniciativa individual para a melhoria de circunstâncias atuais ou a criação de novas situações de trabalho, envolvendo desafio do status quo ao invés da adaptação às condições presentes (Crant, 2000). Esses comportamentos estão relacionados a um conjunto de variáveis antecedentes (Ex.: personalidade proativa, iniciativa pessoal, envolvimento no trabalho, orientação para objetivos) e conseqüentes (Ex.; desempenho no trabalho, sucesso na carreira, atitudes no trabalho). Para esse autor a avaliação do custobenefício do uso de comportamentos pro-ativos no ambiente de trabalho é um fator determinante para o indivíduo fazer uso desses comportamentos. Estratégias para aplicação do aprendido, em sua noção específica, podem ser entendidas como um conjunto de comportamentos pro-ativos, que tem um objetivo específico, a aplicação do aprendido no trabalho. Como Crant define, esses comportamentos são precedidos de um conjunto de cognições e afetos que funcionam como processo decisório para o uso de tais ações.

Uma outra variável, que possui relação com o conceito tema desse estudo, refere-se às estratégias de aprendizagem no ambiente de trabalho. Além da proximidade com o objeto de análise das ações de T\&D, as estratégias de aprendizagem também são estruturadas a partir de três componentes. Warr e Downing (2000) definem essas estratégias como um conjunto de ações, encobertas ou não, de processamento de informação, que tem por objetivo a facilitação do processo de aquisição, armazenamento e evocação de informações a serem aprendidas. Essas estratégias são estruturadas em três componentes: cognitivo, afetivo e comportamental. Os autores 
apresentam vários exemplos do que seriam as estratégias cognitivas, como evocação, organização e elaboração do conteúdo a ser aprendido. No componente afetivo, seriam exemplos de estratégias de aprendizagem o controle emocional e motivacional e, no componente comportamental, seriam exemplos a busca de ajuda com colegas e em material escrito e a aplicação do que foi aprendido.

Warr e Allan (1998) fazem uma revisão do conceito de estratégias de aprendizagem e os autores apontam os componentes desse conceito e suas relações com outras variáveis e com fatores relativos à aplicação do aprendido no ambiente de trabalho, demonstrando que tais estratégias são importantes para predizer aprendizagem no trabalho e em ações educacionais das organizações. Tomando como ponto de partida a mesma lógica de uso do conceito de estratégias de aprendizagem, poder-se-ia compreender que estratégias para aplicação do aprendido é um constructo paralelo, tendo em vista que o conceito de estratégias para aplicação diferencia-se à medida que se torna específico do ambiente pós-treinamento e direcionado para aplicação das competências no trabalho, mas possui semelhanças na sua estruturação teórica, na operacionalização dos componentes e na lógica do uso de partes do conceito (Ex.: o termo estratégia).

Existe um conjunto de variáveis individuais que poderiam ser consideradas como antecedentes das estratégias de aplicação no trabalho do aprendido em treinamento. A auto-eficácia seria uma dessas variáveis, pois à medida que o treinando percebe que possui capacidade de realizar aquilo que se propõe a fazer, ele teria melhores condições para desenvolver e utilizar tais estratégias de aplicação do aprendido. Componentes do modelo de cinco fatores de personalidade também poderiam ser considerados como antecedentes. Segundo P. Warr (comunicação pessoal, 24/03/2003), pessoas com maior nível de Consciensiosidade e Abertura à Experiências tendem a aprender e aplicar mais os conhecimentos no ambiente de trabalho. Em uma meta-análise das relações entre os cinco fatores de personalidade e indicadores de desempenho no trabalho, Barrick e Mount (1991) apontam que pessoas com maiores graus de extroversão, consciensiosidade e abertura a experiências têm maior proficiência em ações de capacitação nas organizações. Com essas evidências, poder-se-ia hipotetizar que a maior magnitude dessas características de personalidade nos treinandos levaria os mesmos a utilizar mais as estratégias para aplicação do aprendido. Um último antecedente das estratégias pode ser atitudes no trabalho (i.e., satisfação, comprometimento), tanto com relações diretas como com relações moderadoras. Assim, faz-se necessário o aprimoramento dos estudos desse fenômeno, de forma que ele possa ser mais bem compreendido e diferenciado de outros constructos correlatos.

A seguir, é apresentada uma análise conceitual, que se faz necessária para a proposição, definição e delimitação do conceito de estratégias para aplicação do aprendido, baseada nos preceitos da filosofia da linguagem (Ryle, 1949).

\section{Revisão conceitual: Estratégias para aplicação do aprendido}

Como tratado na revisão de literatura deste artigo, a noção teórica de estratégias de enfrentamento é essencial para a compreensão, delimitação e estudo do fenômeno de estratégias para aplicação do aprendido (ver Marx, 1982). Isso se faz necessário devido à importância do desenvolvimento e utilização de tais ações de enfrentamento no ambiente pós-treinamento, para a criação de condições necessárias de aplicação, além da manutenção dos comportamentos de aplicação. Por esse motivo, a revisão conceitual se inicia pela explicitação da lógica do uso do conceito de coping (estratégias de enfrentamento) e, na seqüência, das relações conceituais de estratégias para aplicação com metas, comportamentos pro-ativos e estratégias de aprendizagem.

$\mathrm{Na}$ linguagem técnica em psicologia, o conceito de coping é traduzido, para a língua portuguesa, como estratégias de enfrentamento ${ }^{3}$. Notoriamente atrelada aos estudos de estresse, a noção de coping é entendida como um conjunto de estratégias utilizadas pelas pessoas para adaptarem-se a circunstâncias adversas. Observa-se na literatura especializada que essa noção teórica é compreendida de duas formas. Segundo Antoniazzi, Dell'Aglio e Bandeira (1998), a primeira conceituação de estratégias de enfrentamentorelaciona essas a estilos pessoais de enfrentar situações adversas, sendo, então, uma característica disposicional do indivíduo, ligada à noção de personalidade. Nesta perspectiva, a estratégia de enfrentamento poderia ser entendida como uma característica disposicional fechada, exatamente como a noção apresentada por Ryle (1949). Segundo Antoniazzi e colaboradores, a outra abordagem de estratégia de enfrentamento é processual, onde estas são referentes a um conjunto de cognições e comportamentos tomados no curso de um episódio particular de estresse. Assim, as estratégia de enfrentamento são concebidas como associadas a fatores situacionais desencadeadores.

$\mathrm{Na}$ linguagem coloquial (língua inglesa), o termo coping é derivado do verbo cope que significa, segundo Collins-Cobuild (1995), reconhecer e direcionar esforços para uma situação e ter êxito. Caso a situação seja desagradável o indivíduo pode confrontá-la ou conformar-se com ela. Neste sentido, o uso do verbo cope exige um acompanhamento, para possibilitar a identificação do objeto da ação enfrentar. Desta forma, o verbo cope não pode ser considerado um verbo de realização, pois este não implicaria no resultado final da ação. Se o verbo cope exige um acompanhamento, então ele tem uma função adverbial, pois seu uso isolado não tem sentido lógico. Também faz parte da lógica do uso do verbo "enfrentar" a descrição de uma relação entre eventos. Desta forma, o conceito de estratégias de enfrentamento faz parte da noção de conceito disposicional cunhada por Ryle (1949), sendo possível utilizá-lo para explicar uma proposição relacional contida em uma frase tipo "se..., então...". Também faz parte da lógica desse conceito disposicional o seu caráter aberto, pois ele não especifica um tipo de resultado em particular. Uma tradução para o português do verbo cope poderia ser contender, lutar, competir com alguém ou alguma coisa. $\mathrm{Na}$ tradução proposta, mantem-se a noção de sucesso ou de condição de igualdade. Na língua portuguesa, Holanda (1999) indica que a noção de contender está relacionada a oposição, contraposição, luta, briga, enfrentamento.

\footnotetext{
${ }^{3}$ Os conceitos em análise são destacados.
} 
Com inspiração nas questões empíricas presentes na literatura de T\&D, sobre formas de possibilitar o incremento da transferência de aprendizagem para o ambiente de trabalho, observa-se a necessidade de proposição de um novo conceito. A linha de pesquisa de estratégias para o aumento da transferência desperta a idéia de que existem ações realizadas pelos trabalhadores que possibilitam a transferência de aprendizagem para o trabalho. Assim, estrutura-se uma questão empírica: mesmo na ausência de um treinamento de habilidades específicas para aumentar a transferência, os treinados emitirão comportamentos para aplicação do aprendido? Uma forma de abordar esse tema passa por uma questão conceitual: a revisão do conceito de coping, propondo sua ampliação e transposição para o campo de estudos de T\&D. Como proposta conceitual para descrever o fenômeno é que se apresenta a definição de estratégias para aplicação no trabalho do aprendido em treinamento.

Este conceito é entendido como contexto dependente e definese como um conjunto de cognições, afetos e ações dirigidas para criar condições necessárias, no ambiente de trabalho, para a aplicação das competências desenvolvidas em situação de treinamento. A ampliação conceitual, em relação a coping, ocorre à medida que a noção de estratégias para aplicação não está restrita ao enfrentamento de situações adversas. Dessa forma, o conceito é ampliado uma vez que a) ele não se refere apenas a situações adversas; e, b) seu uso será restrito aos ambientes organizacionais pós-treinamento, pois diz respeito a um fenômeno situado e específico.

Uma outra característica desse conceito é a sua estreita relação com metas. O termo estratégias está associado à noção de trilhar ações para atingir um resultado específico, planejado, visualizado. Neste sentido é que a vinculação a metas é explícita e inerente ao conceito. Assim, um componente essencial do conceito são as metas, como definidas por Locke e colaboradores (1981), uma vez que parte do processo de formulação das estratégias passa pela definição de metas para aplicação do aprendido.

Um dos pontos centrais da definição é o verbo aplicar. A exemplo do verbo enfrentar, aplicar não é de realização e tem uma característica aberta. Aparentemente, aplicar também possui uma função de descrição da relação entre eventos. Aplicar carrega consigo a necessidade de complementação, pois é ilógico seu uso isolado. Faz-se, assim, necessária a associação de aplicar a aprendido, dando função ao verbo central do conceito. Exatamente por esta razão é que o verbo aplicar, como ocorre com coping, tem uma característica adverbial, pois ele necessita de um objeto para ter sentido lógico.

Aplicando-se o princípio polar a esse conceito, não seriam estratégias para aplicação do aprendido: cognições e ações que não fossem direcionadas para possibilitar a aplicação do aprendido como atos para diminuição de estresse no trabalho, resultados associados ao uso do aprendido no trabalho, os efeitos do uso das novas competências no desempenho no trabalho, entre outros. Seriam exemplos de estratégias para aplicação do aprendido o reconhecimento de situações adversas para aplicação do aprendido no trabalho, a busca pela definição de metas para aplicação do aprendido, entre outros.

Como já foi salientado anteriormente, esse conceito tem uma estrutura teórica similar àquela apresentada por Warr e Downing (2000) para o constructo de estratégias de aprendizagem e também de comportamentos pro-ativos, como definido por Crant (2000). Para o uso das estratégias de aplicação no trabalho do aprendido em treinamento, é fundamental que o treinado faça a leitura desse ambiente para selecionar a estratégia mais adequada, visando a gerar a condição necessária da aplicação, ou, até, definir se o uso da estratégia para aplicação faz sentido no ambiente organizacional. Nessa perspectiva é que o componente cognitivo do conceito exerce seu papel explicativo. $\mathrm{O}$ componente afetivo funciona como um mecanismo de manutenção do uso de estratégias para aplicação do aprendido. Já o componente comportamental se resume à ação de modificação do ambiente (aqui entendido como físico e social) para a criação da condição necessária à aplicação. Dessa forma, o constructo possui uma estrutura tripartite, composta por cognições, comportamentos e afetos.

Os objetivos desse estudo são de apresentar uma revisão conceitual e da literatura sobre estratégias para aplicação do aprendido no trabalho e de desenvolver e validar uma escala para mensuração de estratégias para aplicação do aprendido em uma amostra de trabalhadores brasileiros. O primeiro objetivo já foi alcançado, até este ponto do texto.

\section{Método}

\section{Participantes}

Participaram desse estudo 298 trabalhadores de várias organizações do Distrito Federal. Do total, 51\% eram do sexo masculino. Metade da amostra (50\%) tinha ao menos o ensino médio completo, seguido de curso superior completo (32\%). A média de idade dos participantes foi de 31,41 anos ( $d p=10,24)$. O tempo médio de experiência profissional foi de 12,03 anos $(d p=9,25)$. Os participantes relataram uma experiência média de 11,13 treinamentos realizados durante sua vida profissional ( $d p=14,65)$. Sobre essa última questão é importante ressaltar que nenhum participante relatou que não teve experiência com treinamentos. Essa variável indica que existe uma grande variação nos relatos da amostra sobre o número de treinamentos realizados, mas, de forma geral, a grande maioria tem relativa experiência com treinamentos. Vale ressaltar que esse indicador é essencial para a presente pesquisa, uma vez que dá indícios sobre as oportunidades de desenvolvimento das estratégias para aplicação do aprendido.

\section{Instrumentos}

Foi utilizado um questionário composto de duas partes. Uma delas incluía cinco questões sócio-demográficas (i.e., sexo, idade, escolaridade, tempo de experiência profissional e número de treinamentos realizados durante a vida profissional). Essas questões tinham como função descrever e segmentar a amostra, visando à comparação entre diferentes grupos.

A outra parte do questionário era composta por 25 questões, associadas a uma escala Likert de 7 pontos de freqüência, desenvolvidas para mensuração do constructo proposto e anteriormente definido. Esses itens foram desenvolvidos a partir de 13 entrevistas utilizando a técnica de incidentes críticos, com profissionais de três organizações diferentes. Essas entrevistas geraram 
mais de 100 indicadores, que subsidiaram a construção de 27 itens que supostamente representavam indicadores do mencionado constructo. Após uma validação semântica com um grupo de especialistas em modelos de avaliação de treinamento, foi sugerida a retirada de três itens. Depois da análise de especialistas em construção de escalas, foi sugerida a inserção de um novo item. Ao final, o instrumento aplicado na amostra ficou composto por 25 itens. Esses itens foram organizados em três fatores hipotéticos distintos: (F1) cognições de avaliação do ambiente pós-treinamento para a identificação de fatores restritivos à aplicação do aprendido, (F2) afetos relativos à manutenção da aplicação do aprendido e (F3) comportamentos voltados para a criação de condições para a aplicação do aprendido no trabalho.

$\mathrm{O}$ questionário foi aplicado de duas diferentes formas. $\mathrm{Na}$ primeira, os pesquisadores convidaram alunos de cursos superiores e de especialização noturnos (quatro instituições de ensino distintas) para participar da pesquisa. Nesse caso a coleta era realizada na sala de aula e garantiu-se, por meio de instrução verbal e conferência na tabulação dos dados, que apenas pessoas com experiência profissional fizessem parte da amostra da pesquisa. A grande maioria da amostra tinha experiência profissional, uma vez que estudantes de nível superior noturno freqüentemente se envolvem em atividades laborais durante o dia. A segunda estratégia utilizada foi a aplicação coletiva do questionário em situações de reunião em organizações de trabalho. Nesse caso a estratégia também foi coletiva. Com esse procedimento de coleta de dados garantiu-se uma grande variabilidade de vinculações organizacionais dos participantes. Para procurar preservar o anonimato dos participantes, optou-se por não coletar informações sobre suas organizações de trabalho.

Para atingir os objetivos dessa pesquisa foram realizadas análises exploratórias dos dados, para adequação ao modelo linear geral (Tabachnick \& Fidell, 2001). As análises principais realizadas, para testar a estrutura empírica da escala, foram a dos componentes principais, fatorial com método de fatoração dos eixos principais e rotação oblíqua e paralela para determinação do número de fatores (Horn, 1965; Zwick \& Velicer, 1986).

\section{Resultados}

As análises exploratórias de dados apontaram que o número de casos omissos era insignificante, pois apenas em um item esse valor era próximo a $2 \%$. Devido a essa característica, optou-se pela substituição dos valores omissos pela média geral. De todos os itens, a análise exploratória indicou que 13 possuem valores altos e significativos de assimetria e seis possuem essas características em achatamento. Mas apenas três itens possuem valores significativos em ambos os indicadores, o que levou à decisão de não realizar-se manipulações das variáveis para aproximá-las de valores mais simétricos. A inspeção da matriz de correlações aponta que mais da metade possuem valores superiores a 0,30, o que indica a adequação da mesma para a utilização de técnicas multivariadas. A partir dessa inspeção não foram detectados problemas de singularidade e multicolinearidade. A análise também indicou a existência de 14 casos extremos multivariados (critério de $\alpha=0,001$ da distribuição $\chi^{2}$ na distância de Mahalanobis). Devido ao pequeno valor e ao fato desses casos extremos serem influentes na solução, optou-se pela exclusão dos mesmos do banco de dados. Dessa forma, as análises principais foram realizadas com 284 casos.

A análise de componentes principais apontou que a matriz é fatorável $(\mathrm{KMO}=0,898)$. O valor do determinante da matriz de correlações é muito próximo a zero. Foram selecionados todos os componentes com autovalores superiores a 1. Isso resultou em uma solução de 5 componentes. Mas como esse critério é altamente sujeito a erros de decisão na extração de fatores (Pasquali, 2000; Tabachnick \& Fidell, 2001; Zwick \& Velicer, 1986), foram utilizadas outras estratégias para a determinação do número de fatores a serem extraídos na análise seguinte. Um

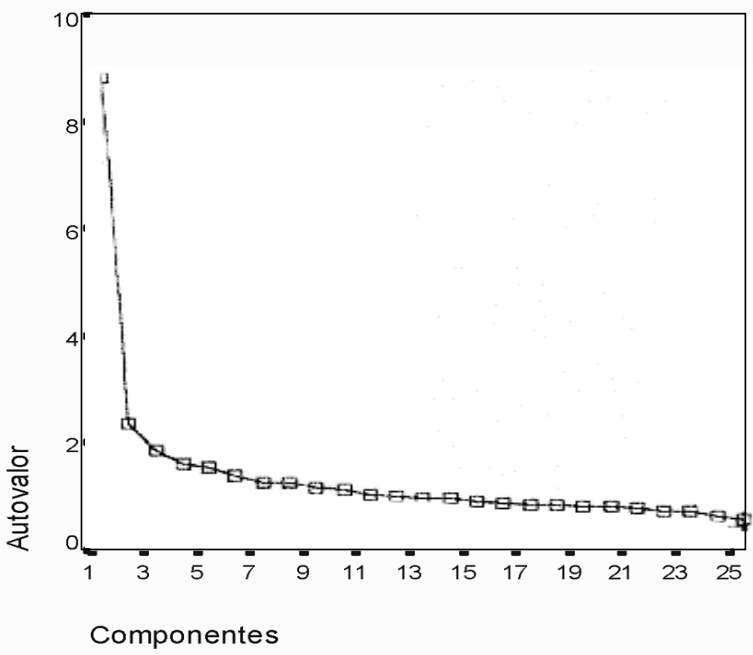

Figura 1. Gráfico de sedimentação dos autovalores, obtido na análise dos componentes principais realizada com os 25 itens da escala.

dos critérios foi a inspeção do gráfico de sedimentação, apresentado na Figura 1.

Como pode ser observada na Figura 1, a curva de sedimentação passa a ter uma queda gradual após o segundo componente. Dessa forma, a extração deveria ser feita em dois fatores. Uma outra estratégia utilizada para a extração de fatores foi a análise paralela (Zwick \& Velicer, 1986), realizada em um banco de dados de mesmo tamanho, mas gerado aleatoriamente. Essa análise indicou que deveriam ser extraídos três fatores. Seguindo a recomendação dos dois testes apresentados, decidiu-se pela extração de dois e três fatores na análise fatorial, sendo o critério teórico de análise dos itens o determinante da estrutura empírica final da escala.

Como estratégia de aproveitar apenas os itens com melhor relação com o fator, decidiu-se pela manutenção daqueles com carga fatorial mínima de 0,45. Essa decisão mais conservadora tem como justificativa a necessidade de uma estrutura empírica enxuta e o aproveitamento dos melhores descritores da estrutura latente.

A análise de fatoração dos eixos principais com extração de três fatores, como indicado pela análise paralela, mostrou-se conceitualmente confusa, efetivando uma agregação inconsistente de dois itens que se ajustam em um pequeno fator, com justificativa 
Tabela 1

Itens, Cargas Fatoriais, Comunalidades e Índices de Consistência Interna da Solução Fatorial da Escala.

\begin{tabular}{|c|c|c|c|}
\hline Itens & $\begin{array}{l}\text { Fator } 1 \\
\alpha=0,88\end{array}$ & $\begin{array}{l}\text { Fator } 2 \\
\alpha=0,83\end{array}$ & $b^{2}$ \\
\hline $\begin{array}{l}\text { 18. Procuro convencer meu chefe que para utilizar o aprendido deve- } \\
\text { se designar um tempo de dedicação. }\end{array}$ & 0,865 & & 0,607 \\
\hline $\begin{array}{l}\text { 19. Negocio com meu chefe o tempo necessário (horas por semana) } \\
\text { que dedicarei para aplicar o aprendido. }\end{array}$ & 0,708 & & 0,463 \\
\hline $\begin{array}{l}\text { 25. Solicito que minha chefia/supervisão imediata defina metas para } \\
\text { aplicação do que aprendi em treinamento. }\end{array}$ & 0,683 & & 0,426 \\
\hline $\begin{array}{l}\text { 23. Solicito a aquisição de material/equipamentos, necessários para a } \\
\text { aplicação do que aprendi. }\end{array}$ & 0,678 & & 0,478 \\
\hline $\begin{array}{l}\text { 24. Solicito os recursos necessários (ex: financeiros, materiais, } \\
\text { humanos) para aplicar o que aprendi. }\end{array}$ & 0,672 & & 0,515 \\
\hline $\begin{array}{l}\text { 14. Mostro para minha chefia/supervisão as vantagens em adquirir } \\
\text { equipamentos que possibilitem a aplicação do que aprendi. }\end{array}$ & 0,621 & & 0,494 \\
\hline $\begin{array}{l}\text { 16. Mostro para minha chefia/supervisão quais as vantagens da } \\
\text { aplicação, no trabalho, do que aprendi em treinamento. }\end{array}$ & 0,580 & & 0,482 \\
\hline $\begin{array}{l}\text { 17. Mostro para os meus colegas de trabalho quais os benefícios de } \\
\text { utilizar o que aprendi em treinamento. }\end{array}$ & 0,514 & & 0,464 \\
\hline 20. Defino metas fáceis para aplicar o que aprendi em treinamento. & 0,480 & & 0,215 \\
\hline $\begin{array}{l}\text { 21. Peço orientações a colegas mais experientes para aplicar o que } \\
\text { aprendi em treinamento. }\end{array}$ & 0,453 & & 0,337 \\
\hline $\begin{array}{l}\text { 12.Informo minha chefia/supervisão sobre o que aprendi em } \\
\text { treinamento, quando retorno ao trabalho (ou termino o treinamento). }\end{array}$ & & & 0,329 \\
\hline 22. Preocupo-me quando é difícil aplicar, no trabalho, o que aprendi. & & & 0,237 \\
\hline $\begin{array}{l}\text { 10. Identifico as dificuldades que encontro no ambiente de trabalho } \\
\text { para aplicar o que aprendi. }\end{array}$ & & 0,711 & 0,418 \\
\hline $\begin{array}{l}\text { 1. Acredito que é possível aplicar no trabalho o que aprendi em } \\
\text { treinamento. }\end{array}$ & & 0,612 & 0,325 \\
\hline 7. Busco as informações necessárias para aplicar o que aprendi. & & 0,596 & 0,520 \\
\hline 6. Avalio como estou aplicando no trabalho o que aprendi em treinamento. & & 0,584 & 0,482 \\
\hline $\begin{array}{l}\text { 2. Identifico antecipadamente as situações que podem dificultar a } \\
\text { aplicação do que aprendi em treinamento. }\end{array}$ & & 0,571 & 0,258 \\
\hline $\begin{array}{l}\text { 11. É muito importante aplicar no trabalho o que aprendi em } \\
\text { treinamento. }\end{array}$ & & 0,525 & 0,308 \\
\hline $\begin{array}{l}\text { 4. Admiro as pessoas que conseguem aplicar no trabalho o que } \\
\text { aprenderam em treinamento. }\end{array}$ & & 0,500 & 0,271 \\
\hline $\begin{array}{l}\text { 8. Consulto material de treinamento para aplicar, no trabalho, o que } \\
\text { aprendi em treinamento. }\end{array}$ & & 0,488 & 0,417 \\
\hline $\begin{array}{l}\text { 5. Apresento resultados da aplicação do que aprendi para meus } \\
\text { colegas e chefe/supervisor. }\end{array}$ & & 0,460 & 0,415 \\
\hline 15. Fico feliz quando consigo aplicar no trabalho o que aprendi. & & & 0,284 \\
\hline 9. Defino metas difíceis para aplicar o que aprendi em treinamento. & & & 0,222 \\
\hline $\begin{array}{l}\text { 13. Modifico a forma de fazer meu trabalho para aplicar o que aprendi } \\
\text { em treinamento. }\end{array}$ & & & 0,326 \\
\hline 3. Aplico, em conjunto com meus colegas, o que aprendi em treinamento. & & & 0,262 \\
\hline
\end{tabular}

teórica nula. Por esse motivo, optou-se pela extração de dois fatores como estrutura empírica definitiva nesse trabalho.

A análise com extração de dois fatores apresentou uma estrutura com boa justificativa teórica. O primeiro fator era correspondente a ações de criação de condições para aplicação do aprendido no ambiente de trabalho e outro com itens referentes a cognições e afetos relacionados à aplicação do aprendido. Os indicadores dos dois fatores, bem como seus descritores de conteúdo, são apresentados na Tabela 1.
Como pode ser observado na Tabela 1, foram eliminados da solução fatorial seis itens, com critério de carga fatorial igual ou superior a 0,45 . Os indicadores de consistência interna dos fatores são muito bons (superiores a 0,85 ) e a variação das comunalidades dos itens é adequada para a escala, indicando que há relação de magnitude significativa entre cada um dos itens com o conjunto total.

É importante ressaltar que três itens possuem uma possível divergência teórica da hipótese inicial do estudo, pois se agregaram em fatores diferentes. O primeiro é o item 20 ("Defino metas difíceis 
para aplicação do aprendido"), que obteve carga significativa no fator 1. Isso pode ser devido à percepção dos respondentes de que a definição de metas é uma ação sobre o ambiente e não uma avaliação do mesmo, como foi hipotetizado inicialmente. Os outros dois casos correspondem, respectivamente, aos itens 8 ("Consulto material de treinamento...") e 5 (“Apresento resultados da aplicação..."), que obtiveram carga fatorial no fator 2 . Inicialmente esses dois itens foram hipotetizados como ações de modificação do ambiente, mas o padrão de covariação desses itens, nessa amostra, os aproxima mais de cognições e afetos de manutenção dos comportamentos de criação de condições de aplicação. Isso pode ser devido a uma compreensão de que apresentar resultados e consulta a material sejam ações anteriores à criação de condições de aplicação.

\section{Discussão}

O constructo proposto pode ser considerado um fenômeno compartilhado pelos respondentes da escala. A estrutura teórica obteve corroboração, ainda que houve a perda de oito itens, graças ao critério conservador de valor de corte da carga fatorial, e a melhor solução com dois fatores. Essa última característica não é uma questão perturbadora, tendo em vista que foi observada uma agregação em um único fator de dois fatores hipotetizados. Assim, a estrutura empírica final é mais parcimoniosa e representa a estrutura teórica desenvolvida para o instrumento de mensuração.

O primeiro fator da solução corrobora um conjunto de indicadores de comportamentos de prevenção de recaídas, como apresentado por Marx (1982). Já o segundo fator também traz uma série de elementos de ações de identificação das dificuldades encontradas no ambiente de trabalho posterior ao treinamento, elemento essencial do modelo daquele autor. Dentro da solução empírica da escala, também são observados itens que representam elementos do modelo de estabelecimento de metas, que já foi testado com sucesso por alguns pesquisadores (Ex.: Gist \& cols., 1991; Wexley \& Baldwin, 1986).

Dessa forma os estudos na área de ações para aumentar a transferência do aprendido caminham de forma certeira para proporcionar o aumento da efetividade dos programas de treinamento, uma vez que as pessoas, mesmo sem instrução formal a respeito, tendem a perceber que tais ações possibilitam a aplicação do aprendido. Mas é importante ressaltar que há um conjunto de comportamentos de criação de condições de aplicação, que dizem respeito à dimensão social (i.e., o convencimento da chefia, o envolvimento de colegas de trabalho, entre outros) que não são explicitamente trabalhados nos modelos de prevenção de recaídas e estabelecimento de metas. Aparentemente esses dois modelos são sustentados em um viés de que o indivíduo é um agente direto da transformação do ambiente, tendo em vista que não há uma alusão específica de ação sobre pares e supervisores. Esse resultado aponta para a adequação do estudo desse fenômeno a partir da perspectiva de que os treinandos desenvolvem, independentemente da instrução formal, estratégias para aplicação do aprendido. Além disso, esses resultados ressaltam a importância da ação de modificação de atitudes de pares e chefia para a criação de condições ambientais favoráveis à aplicação. Assim, esse estudo aprimora a compreensão do fenômeno e fornece indicações de novas questões a serem inseridas nos modelos de aumento de transferência de aprendizagem no trabalho.

De forma geral, o grande diferencial proposto nesse trabalho se centra no fato de que todas essas estratégias não foram previamente desenvolvidas nos treinandos. Nessa linha de raciocínio, esse constructo possui uma grande relação com os comportamentos proativos nas organizações de trabalho (Crant, 2000). A corroboração da estrutura teórica sustenta a hipótese de que as pessoas desenvolvem estratégias, que seriam ações proativas, para aplicarem no trabalho o aprendido em treinamentos independentemente de uma instrução formal com tal objetivo. Provavelmente o principal motivo que leva ao desenvolvimento de tais estratégias é relativo à experiência pessoal de cada treinando com situações de retorno ao trabalho após o treinamento, aliado a um conjunto de fatores individuais e situacionais. Seriam fatores individuais que possibilitariam o desenvolvimento de tais estratégias: os valores instrumentais do treinamento para a vida profissional, aliado a uma atitude positiva em frente ao conteúdo do treinamento. Fatores situacionais relacionados ao desenvolvimento de tais estratégias estariam ligados a um ambiente de trabalho que fornecesse abertura para o desenvolvimento de tais estratégias, onde estariam ausentes conseqüências negativas associadas ao uso do aprendido.

Mais estudos devem ser realizados para se compreender como fatores situacionais e individuais determinam o uso das estratégias de aplicação. Modelos com relações de moderação e mediação devem testar o conjunto de preditores das estratégias de aplicação. Outro estudo que se faz necessário é o concernente à relação temporal entre treinamento e oportunidade de aplicação após treinamento e sua interação com o sucesso no uso das estratégias (i.e., a aplicação do aprendido propriamente dita) para se descrever como o papel de fatores situacionais pode condicionar o uso e a ausência do uso das estratégias.

Com essas primeiras evidências empíricas, pode-se esperar que esse constructo seja útil para compreender os fatores que levam ao impacto do treinamento no trabalho dos treinados. Futuras pesquisas devem procurar testar a validade discriminante da medida aqui desenvolvida, em relação a outras medidas dos modelos de avaliação de treinamento, tais como as de suporte à transferência, bem como a medidas de constructos auto-referentes, tais como motivação para aprender, auto-conceito, auto-eficácia e comprometimento organizacional e com a carreira. Também é fundamental incluir essa variável como antecedente de transferência de aprendizagem para o trabalho e de impacto do treinamento no trabalho. Ainda seria útil o desenvolvimento de estudos que busquem compreender os antecedentes das estratégias para aplicação no trabalho do aprendido em treinamento. Com essa última linha de pesquisa, seria possível desenvolver tecnologias para capacitar os trabalhadores no desenvolvimento de estratégias, bem como na criação de um ambiente de trabalho que facilitasse o uso de tais estratégias.

Dessa forma, esse trabalho poderá ter conseqüências para a pesquisa na área de avaliação de treinamento, uma vez que traz 
evidências sobre um novo constructo teórico que pode incrementar os modelos descritivos de impacto do treinamento no trabalho. Esse estudo também traz conseqüências potenciais para os profissionais de recursos humanos das organizações brasileiras, pois abre espaço conceitual e instrumental para o desenvolvimento de futuras tecnologias que poderão ser utilizadas visando aumentar a efetividade das ações de educação nas organizações.

\section{Referências}

Abbad, G. (1999). Um modelo integrado de avaliação de impacto de treinamento no trabalho. Tese de Doutorado não-publicada, Instituto de Psicologia, Universidade de Brasília. Brasilia, DF.

Abbad, G., Borges-Andrade, J. E., Sallorenzo, L. H., Gama, A. L. G. \& Morandini, D. C. (2001). Projeto de treinamento, aprendizagem, satisfação com o treinamento e auto-avaliação de impacto do treinamento no trabalho. Psicologia, Organizações e Trabalho, 1, 129-161.

Abbad, G., Gama, A. L. G. \& Borges-Andrade, J. E. (2000). Treinamento: Análise do relacionamento da avaliação nos níveis de reação, aprendizagem e impacto no trabalho. Revista de Administração Contemporânea, 4, 25-45.

Abbad, G., Pilati, R. \& Pantoja, M. J. (2003). Avaliação de treinamento: Análise da literatura e agenda de pesquisa. Revista de Adminstração da USP, 38, 205-218.

Antoniazzi, A. S., Dell'Aglio, D. D. \& Bandeira, D. R. (1998). O conceito de coping: Uma revisão teórica. Estudos de Psicologia, 3, 273-294.

Barrick, M. R. \& Mount, M. K. (1991). The big five personality dimensions and job performance: A meta-analysis. Personnel Psychology, 44, 1-26.

Borges-Andrade, J. E. (1982). Avaliação somativa de sistemas instrucionais: Integração de três propostas. Tecnologia Educacional, 11, 29-39.

Borges-Andrade, J. E. \& Abbad-OC, G. (1996). Treinamento e desenvolvimento: Reflexões sobre suas pesquisas. Revista de Administração da USP, 31, 112-125.

Britto, M. J. P., Lima, S. M. V. \& Borges-Andrade, J. E. (2001). Avaliação de impacto de treinamento na área de reabilitação: Preditores individuais e situacionais. Revista de Administracão da USP, 36, 46-56.

Burke, L. (1997). Improving positive transfer: A test of relapse prevention training on training outcomes. Human Resource Development Quarterly, 8, 115-128.

Collins (1995). Cobuild English Dictionary. London: Harper Collins.

Colquitt, J. A., LePine, J. A. \& Noe, R. A. (2000). Toward an integrative theory of training motivation: A meta-analytic path analysis of 20 years of research. Journal of Applied Psychology, 83, 654-665.

Crant, J. M. (2000). Proactive behavior in organizations. Journal of Management, 26, 435-462.

Gist, M. E., Stevens, C. K. \& Baveta, A. G. (1991). Effects of self-efficacy and posttraining intervention on the acquisition and maintenance of complex interpersonal skills. Personnel Psychology, 44, 837-861.

Goldstein, I. L. (1993). Training in organizations. Pacific Grove, CA: Brooks/Cole.

Holanda, A. (1999). Dicionário Aurélio Século XXI. São Paulo: Nova Fronteira.

Holton III, E. F., Bates, R. A., Seyler, D. L. \& Carvalho, M. B. (1997). Toward construct validation of a transfer climate instrument. Human Resource Development Quartely, 8, 95-113.

Horn, J. L. (1965). A rationale and test for the number of factors in factor analysis. Psychometrika, 30, 179-185.
Lacerda, E. M. (2002). Motivação, valor instrumental do treinamento, reação, suporte à transferencia e impacto do treinamento no trabalho. Dissertação de Mestrado nãopublicada, Instituto de Psicologia, Universidade de Brasília. Brasília, DF.

Lima, S. M. V., Borges-Andrade, J. E. \& Vieira, S. B. A. (1989). Cursos de curta duração e desempenho em instituições de pesquisa agrícola. Revista de Administração da USP, 24, 36-46.

Locke, E. A., Shaw, K. N., Saari, L. M. \& Latham, G. P. (1981). Goal setting and task performance: 1969-1980. Psychological Bulletin, 90, 125-152.

Marx, R. D. (1982). Relapse prevention of managerial training: A model for maintenance of behavior change. Academy of Management Review, 7, 433-441.

Morin, L. \& Latham, G. P. (2000). The effect of mental practice and goal setting as a transfer of training intervention on supervisor's self-efficacy and communication skills: An exploratory study. Applied Psychology: An Internation Review, 49, 566-578.

Pasquali, L. (2000). Análise fatorial para pesquisadores. Manuscrito não-publicado.

Richman-Hirsch, W. L. (2001). Posttraining interventions to enhance transfer: The moderating effects of work environments. Human Resource Development Quartey, 12, 105-120.

Rouiller, J. Z. \& Goldstein, I. L. (1993). The relationship between organizational transfer climate and positive transfer of training. Human Resource Development Quartely, 4, 377-390.

Ryle, G. (1949). The concept of mind. London: Hutchinson.

Salas, E. \& Cannon-Bowers, J. A. (2001). The science of training. Annual Review of Psychology, 52, 471-499.

Sallorenzo, L. H. (2000). Avaliação de impacto de treinamento no trabalho: Analisando e comparando modelos de predição. Dissertação de Mestrado não-publicada, Instituto de Psicologia, Universidade de Brasília. Brasília, DF.

Stevens, C. K. \& Gist, M. E. (1997). Effects of self-efficacy and goal-orientation training on negotiation skill maintenance: What are the mechanisms? Personnel Psychology, 50, 955-978.

Tabachnick, B. \& Fidell, L. S. (2001). Using multivariate statistics ( $4^{\mathrm{a}}$ ed.). San Francisco, CA: Allyn \& Bacon.

Tannenbaum, S. I., Mathieu, J. E., Salas, E. \& Cannon-Bowers, J. A. (1991). Meeting trainee's expectations: The influence of training fulfillment on the development of commitment, self-efficacy and motivation. Journal of Applied Psychology, 76, 759-769.

Warr, P. \& Allan, C. (1998). Learning strategies and occupactional training. International Review of Industrial and Organizational Psychology, 13, 83-121.

Warr, P. \& Bunce, D. (1995). Trainee characteristics and the outcomes of open learning. Personnel Psychology, 48, 347-375.

Warr, P. \& Downing, J. (2000). Learning strategies, learning anxiety and knowledge acquisition. British Journal of Psychology, 91, 311-333.

Wexley, K. N. \& Baldwin, T. T. (1986). Post-training strategies for facilitating positive transfer: An empirical exploration. Academy of Management Journal, 29, 503-520.

Zwick, W. R. \& Velicer, W. F. (1986). Comparison of five rules for determining the number of components to retain. Psychological Bulletin, 3, 432-442.

Recebido: 29/10/2003

$1^{a}$ Revisão: 16/04/2004

Aceite Final: 30/04/2004

Sobre os autores

Ronaldo Pilati é Doutor em Psicologia. Pesquisador do Instituto de Ciências do Trabalho. Integrante do Grupo PRONEX Treinamento e Comportamento no Trabalho. É Professor do UNICEUB.

Jairo E. Borges-Andrade é Professor Titular do Departamento de Psicologia Social e do Trabalho do Instituto de Psicologia da Universidade de Brasília. É Coordenador do grupo PRONEX Treinamento e Comportamento no Trabalho. 